\title{
Adipogenic Activity of Wild Populations of Rhododendron groenlandicum, a Medicinal Shrub from the James Bay Cree Traditional Pharmacopeia
}

\author{
Michel Rapinski, ${ }^{1,2}$ Lina Musallam, ${ }^{1,3}$ John Thor Arnason, ${ }^{1,4}$ \\ Pierre Haddad, ${ }^{1,3}$ and Alain Cuerrier ${ }^{1,2}$ \\ ${ }^{1}$ Canadian Institutes of Health Research Team in Aboriginal Antidiabetic Medicines, Université de Montréal, Montréal, \\ QC, Canada H3C 3J7 \\ ${ }^{2}$ Institut de Recherche en Biologie Végétale, Jardin Botanique de Montréal, Université de Montréal, 4101 Sherbrooke Est, \\ Montréal, QC, Canada H1X 2B2 \\ ${ }^{3}$ Natural Health Products and Metabolic Diseases Laboratory, Department of Pharmacology, Université de Montréal, \\ Montréal, QC, Canada H3C 3J7 \\ ${ }^{4}$ Centre for Research in Biotechnology and Biopharmaceuticals, Department of Biology, University of Ottawa, Ottawa, \\ ON, Canada K1N 6N5
}

Correspondence should be addressed to Pierre Haddad; pierre.haddad@umontreal.ca and Alain Cuerrier; alain.cuerrier@umontreal.ca

Received 20 March 2015; Accepted 25 May 2015

Academic Editor: Nunziatina De Tommasi

Copyright (c) 2015 Michel Rapinski et al. This is an open access article distributed under the Creative Commons Attribution License, which permits unrestricted use, distribution, and reproduction in any medium, provided the original work is properly cited.

\begin{abstract}
The traditional medicinal plant, Labrador tea (Rhododendron groenlandicum (Oeder) Kron \& Judd; Ericaceae), present in the pharmacopoeia of the Cree of Eeyou Istchee, has shown glitazone-like activity in the 3T3-L1 adipogenesis bioassay. This activity has been attributed to phenolic compounds, which have been shown to vary in this plant as a function of insolation parameters. The goal of this study was to determine if these changes in phenolic content were pharmacologically significant. Leaves were harvested in 2006 throughout the James Bay region of Northern Quebec and ethanol extracts were tested in vitro using the 3T3-L1 murine cell line adipogenesis bioassay. This traditional medicinal plant was found active in the assay. However, there was no detectable spatial pattern in the accumulation of intracellular triglycerides, suggesting that such patterns previously observed in the phenolic profile of Labrador tea were not pharmacologically significant. Nonetheless, a reduction in the adipogenic activity was observed and associated with higher concentrations of quercetin for which selected environmental variables did not appropriately explain its variation.
\end{abstract}

\section{Introduction}

In a previous study on the phytochemistry of the North American medicinal plant, Rhododendron groenlandicum (Oeder) Kron \& Judd (Ericaceae), Labrador tea, we found the concentration of biologically active compounds to vary in Northern Quebec's Hudson and James Bay region [1]. Labrador tea is a common species in Canada's boreal forest. More importantly, it is a popular medicinal plant found in the traditional pharmacopoeia of indigenous populations from the Algonquian, Salish, Wakashan, Tsimshian, and EskimoAleut linguistic families [2-9].
In ethnobotanical studies conducted in six communities of the Cree Nation of Eeyou Istchee (CEI), we found $R$. groenlandicum to be the top-ranked plant species used for the treatment of symptoms associated with type 2 diabetes (T2D) [10-13]. The inherent cultural relevance of this species to CEI traditional medicine (CTM) warrants further investigation into its antidiabetic potential.

The CIHR Team on Antidiabetic Aboriginal Medicines (CIHR-TAAM), formed through collaborative work between CEI communities, the Cree Board of Health and Social Services of James Bay (CBHSSJB), and Canadian academic researchers, has screened many of the multiple plants 
present in the CEI pharmacopoeia [14-17]. Of these, $R$. groenlandicum was shown to possess in vitro glitazone-like activity comparable to rosiglitazone in an adipogenic assay measuring the lipid accumulation in differentiating 3T3-L1 preadipocytes [14].

The antidiabetic drug rosiglitazone induces an increase in the sensitivity to insulin, acting as a PPAR $\gamma$ receptor agonist [18]; the expression of this transcription factor is particularly implicated in the differentiation of adipocytes [19-22] and in insulin sensitivity [18]. Hence, it plays a critical role in the pathogenesis of T2D. The action of PPAR $\gamma$ results in an improvement in the absorption of fatty acids in differentiated adipocytes which store them as triglycerides (TG) [18]. Adipocytes therefore provide storage for fatty substances that would otherwise accumulate in tissues such as skeletal muscle and liver, thereby contributing to metabolic disorders such as insulin resistance [22].

The pharmacological activity of $R$. groenlandicum has been attributed to phenolic compounds [14, 16, 23]. Bioassay-guided fractionation using adipogenesis of 3T3-L1 murine cells confirmed that specific phenolics are the most active compounds [24]. In developing culturally appropriate approaches to treating T2D in the CEI communities, the variation of these compounds in $R$. groenlandicum has important implications in ensuring the quality control of traditional medicinal plants or to develop standardized natural health products (NHPs).

In this study, we assessed possible variations in the antidiabetic potential of $R$. groenlandicum. Our objective was to determine if the phytochemical variations observed in the species' phenolic profile are biologically significant. We evaluated the in vitro pharmacological activity of crude extracts from various localities using the adipogenesis bioassay and hypothesized that high concentrations of phenolic compounds would result in a stronger adipogenic activity.

\section{Materials and Methods}

2.1. Sampling, Extraction, and Phytochemical Analysis. The sampling, extraction, and analytical methods for phytochemical identification and quantification are thoroughly described in Rapinski et al. [1], which reports on the phytochemistry of $R$. groenlandicum. A subsample, selected randomly, of previously reported samples was used in this in vitro study.

Briefly, mature leaves were sampled during the summer of 2006 around the communities of Mistissini, Nemaska, Eastmain, Wemindji, and Whapmagoostui, thus covering much of the northsouth gradient in Eeyou Istchee. Five accessions, each containing leaves from multiple individual plants, were collected within a $50 \mathrm{~km}$ radius around each community and selected for this study. Samples were airdried and preserved in paper bags at room temperature.

Samples were milled through a Wiley Mill at 40 mesh and extracted overnight in $25 \mathrm{~mL} / \mathrm{g}$ of $80 \% \mathrm{EtOH}$ by orbital shaking at room temperature at 250 RPM. The pellet was extracted overnight in $15 \mathrm{~mL} 80 \% \mathrm{EtOH}$. An aliquot $(1 \mathrm{~mL})$ of the pooled supernatants (adjusted to $50.0 \mathrm{~mL}$ in a volumetric flask) was prepared for High Performance
Liquid Chromatography coupled with Diode Array Detector (HPLC-DAD). The leftover crude extracts were dried using a speedVac; the trace water was removed by lyophilization using SuperModulo freeze dryer and the dehydrated extracts were stored at $-80^{\circ} \mathrm{C}$.

Finally, $10 \mu \mathrm{L}$ of each extract aliquot was injected through an autosampler and detected by DAD at $290 \mathrm{~nm}$, bandwidth 4 , reference off. The separations were performed on a Luna C18 column $(250 \times 4.6 \mathrm{~mm}, 5 \mu \mathrm{M}$ particle size $)$. Peak identification was undertaken by cochromatographic comparison of the spectral data adopted in our in-house metabolomics spectral library [23]. A standard curve was constructed by injection of serially diluted marker compounds in methanol. The quantification was based on peak area. The quantitation of putatively identified quercetin-glycosides was achieved based on calibration curve of quercetin-3-galactoside. Each sample was analyzed in triplicate and averaged to account for instrumental variation.

2.2. Cell Culture. 3T3-L1 murine preadipocyte cells were grown to confluence in 24-well plates in DMEM proliferation medium containing $10 \%$ FBS. Media were changed every 2 days. At $24 \mathrm{~h}$ after confluence (day 0 ), cells were induced to differentiate with a short-term differentiation medium of DMEM supplemented with 10\% FBS, $1 \mu \mathrm{M}$ DMX, $250 \mu \mathrm{M}$ IBMX, and $500 \mathrm{nM}$ insulin. After $48 \mathrm{~h}$, the media were replaced with DMEM containing 10\% FBS and $500 \mathrm{nM}$ insulin for long-term differentiation. Cells were differentiated for a total of 5 days with media change every 2 days. Rhododendron groenlandicum crude extracts $(75 \mu \mathrm{g} / \mathrm{mL})$ and rosiglitazone $(10 \mu \mathrm{M}$; positive control) were dissolved in DMSO and added to the cells as of day 0 of differentiation. The final concentration of DMSO was kept at $0.1 \%$ throughout the differentiation period.

2.3. Adipogenesis. We measured intracellular TG content at day 5 of differentiation using the AdipoRed reagent according to the manufacturer's instructions. Methods have been previously described in Spoor et al. [14] and Harbilas et al. [16]. In short, wells containing adipocytes were washed twice with phosphate-buffered saline (PBS) before $1 \mathrm{~mL}$ of PBS containing $30 \mu \mathrm{L}$ of AdipoRed reagent was added to each well and incubated for 15 minutes at room temperature. AdipoRed becomes fluorescent when partitioned in a hydrophobic compartment, namely, intracellular triglycerides (TG). The fluorescence of each well was measured with a Wallac Victor2 fluorimeter (Perkin-Elmer, Saint-Laurent, QC) at an excitation wavelength of $485 \mathrm{~nm}$ and an emission wavelength of $572 \mathrm{~nm}$. The results were reported as percentage of the vehicle control, $0.1 \%$ DMSO.

2.4. Cell Lines, Chemicals, Biochemicals, and Standards. For the identification and quantification of phenolic markers, (+)-catechin (1), chlorogenic acid (2), (-)-epicatechin (3), $p$-coumaric acid (4), rutin (5), quercetin-3-galactoside (6), quercetin-3-glucoside (7), quercetin-3-rhamnoside (12), myricetin (13), and quercetin (14) were purchased from Sigma-Aldrich (Oakville, Ontario, Canada) and Extrasynthese (Genay, France). HPLC grade water, acetonitrile, and 
formic acid (99\% purity) were purchased from SigmaAldrich.

For cell culture and adipogenesis, preadipocyte 3T3-L1 cell line was purchased from the American Type Culture Collection (ATCC; Manassas, VA). Dexamethasone (DMX), bovine pancreatic insulin, 3-isobutyl-1-methylxanthine (IBMX), and Dimethyl sulfoxide (DMSO) were purchased from Sigma-Aldrich (Oakville, ON). Rosiglitazone was obtained from Alexis Biochemicals (Hornby, ON).

Dulbecco's Modified Eagle Medium (DMEM), fetal bovine serum (FBS), and bovine calf serum (NCS) were from Wisent Inc. (Saint-Bruno, QC). AdipoRed reagent was purchased from Cambrex Bio Science Walkersville Inc. (Walkersville, $\mathrm{MD})$.

2.5. Environmental Data. Annual estimates of bioclimatic variables, namely, annual temperature range, corresponding to our sampling year and long-term estimates for insolation variables were provided by the Canadian Forest Services of Natural Resources Canada [25]. Long-term estimates were derived from multidecade meteorological data collected from 1971 to $2000[26,27]$.

2.6. Statistical Analysis. To reduce interassay variation, TG content was normalized relative to each assay's vehicle control, $0.1 \%$ DMSO, set at $100 \%$. Rhododendron groenlandicum and rosiglitazone always induced significant increases in activity as verified by the fact that the $95 \%$ confidence interval of the mean activity (quadruplicate determinations) did not include the $100 \%$ adipogenic activity reference $(p<$ 0.05 ). Differences between communities were analyzed by one-way analysis of variance. The relationships between TG content and compounds were analyzed by multiple and simple linear regressions. To represent the adipogenic activity of R. groenlandicum and the quantified compounds, principal components analysis (PCA) was performed on the matrix of these compounds using the correlation matrix. Individual samples were scored onto the PCA axes and represented with the vectors for each compounds. TG content was subsequently projected as a supplementary variable onto the principal components in order to interpret the dimensions of variability. In doing so, the calculation of distances between each of the samples and the construction principal components depends only on their phytochemical profile. Using the transition formulae described by Lê et al. [28], the coordinates for TG content are calculated using the original eigenvalues. Finally, we partitioned the variation in the adipogenic activity of $R$. groenlandicum between the two sets of variables: compounds and environmental factors. This was done using a partial-redundancy analysis (partial$\mathrm{RDA}$ ) approach $[29,30]$. All analyses were performed using $\mathrm{R}$ statistical language [31]. Results are reported as means $\pm \mathrm{SD}$ and statistical significance is set at $\alpha=0.05$.

\section{Results and Discussion}

The phytochemistry of the same R. groenlandicum accessions has already been described and discussed in greater length in

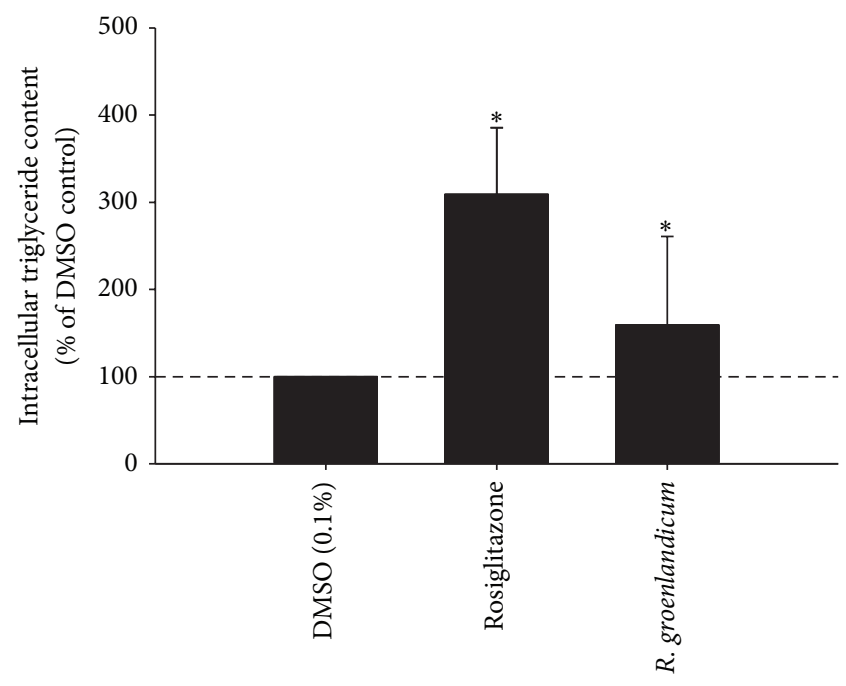

FIGURE 1: Effect of R. groenlandicum crude leaf extracts from Northern Quebec on lipid accumulation. Intracellular triglyceride content was measured by AdipoRed fluorescence, in live 3T3-L1 murine adipocytes incubated with plant extracts for 5 days after differentiation. Means $\pm \mathrm{SD}(n=4$ for rosiglitazone, $n=100$ for $R$. groenlandicum) are normalized to the vehicle control $(0.1 \%$ DMSO). Asterisk $(*)$ indicates significant differences with respect to the DMSO control at $\alpha=0.05$.

Rapinski et al. [1]. Here, we present the results of a subsample of 2006 accessions in the adipogenesis bioassay.

The glitazone-like activity of $R$. groenlandicum to increase the accumulation of intracellular TG in 3T3-L1 adipocytes was measured at day 5 of differentiation. Extracts increased adipogenesis, with an average content of TG of $159.0 \%$ that of DMSO (Figure 1) and a 95\% confidence interval of 138.8$179.1 \%$ of DMSO. The adipogenic activity of $R$. groenlandicum was roughly half of the positive control, rosiglitazone. This is lower than what has previously been reported for this species. Spoor et al. [14] reported the stimulation of adipogenesis to be comparable to rosiglitazone, while later determinations measured an activity representing two-thirds that of the antidiabetic drug [24]. With few exceptions (Figure 2), our results nonetheless confirm the adipogenic potential of this species. It is important to consider the fact that previous determinations of activity were carried out using extracts prepared from large quantities of source material (large number of individual plants) collected a few years prior to the material used in the present studies. Hence, interindividual variations were absent and different climatic conditions may have prevailed. This can explain, at least in part, the differences in adipogenic potential observed between the studies.

There were no statistically distinct spatial patterns detected in the pharmacological activity of $R$. groenlandicum. None of the communities sampled possessed accessions which significantly increased intracellular TG more than the others ( $p=0.348$, Figure 3$)$. We have previously found that biologically active phenolics were greater in collections made around the communities of Nemaska, Eastmain, and Wemindji [1]. The adipogenic activity of $R$. groenlandicum 


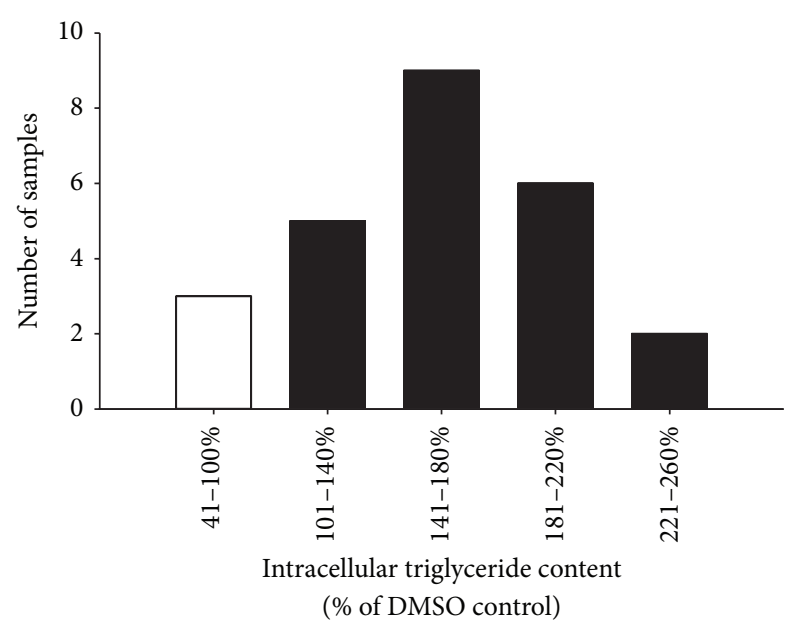

FIGURE 2: Frequency distribution of the adipogenic activity from 25 samples of $R$. groenlandicum leaves collected throughout Northern Quebec. Intracellular triglyceride content was measured by AdipoRed fluorescence, in live 3T3-L1 murine adipocytes incubated with plant extracts for 5 days after differentiation. Triglyceride content was normalized to the vehicle control (0.1\% DMSO). Samples with content levels below $100 \%$ (in white) were considered inhibitory and decreased lipid accumulation.

followed a similar trend, as can be observed in Figure 3, although statistical significance of a polynomial relationship was not achieved ( $p=0.170)$, possibly due to high variability. This suggests that variations in the phytochemical profiles, observed in Rapinski et al. [1], may be pharmacologically relevant, but further studies will be necessary to confirm this point.

Indeed, we found that quantified compounds explained considerable variability obtained in this species' pharmacological activity $\left(p=0.0279, R_{\mathrm{adj}}^{2}=0.491\right)$. The distribution of $R$. groenlandicum samples based on their phytochemical profile was reconstructed into a reduced three-dimensional space, which represented $69.92 \%$ of the samples' variation over three statistically constructed principal components, or axes (Figure 4). Each principal component, from the first to the third, respectively, explained $35.99 \%\left(\lambda_{1}=3.96\right), 22.97 \%$ $\left(\lambda_{2}=2.53\right)$, and $10.96 \%\left(\lambda_{3}=1.20\right)$ of the variation. The direction and proximity of arrows for some major markers suggest that these are highly correlated (Figure 4). When projecting intracellular TG onto this plot, it did not appear to be well correlated with the bulk of these markers, many of which were found near a $90^{\circ}$ angle from it, thus indicating week or null correlations. One of the only markers for which a significant relationship with TG appears to exist is quercetin (Figures 4(b) and 4(c)). This suggests that, out of all the compounds assessed, variations in the adipogenic activity of $R$. groenlandicum are most vulnerable to changes in the content of quercetin found in the crude extracts. Figure 5 further illustrates the linear correlation $\left(p=0.0458, R^{2}=\right.$ 0.162 ) whereby the adipogenic activity of $R$. groenlandicum decreases as the concentration of quercetin in the sample increases. This is consistent with observations from our own group [24] where pure quercetin was found to inhibit

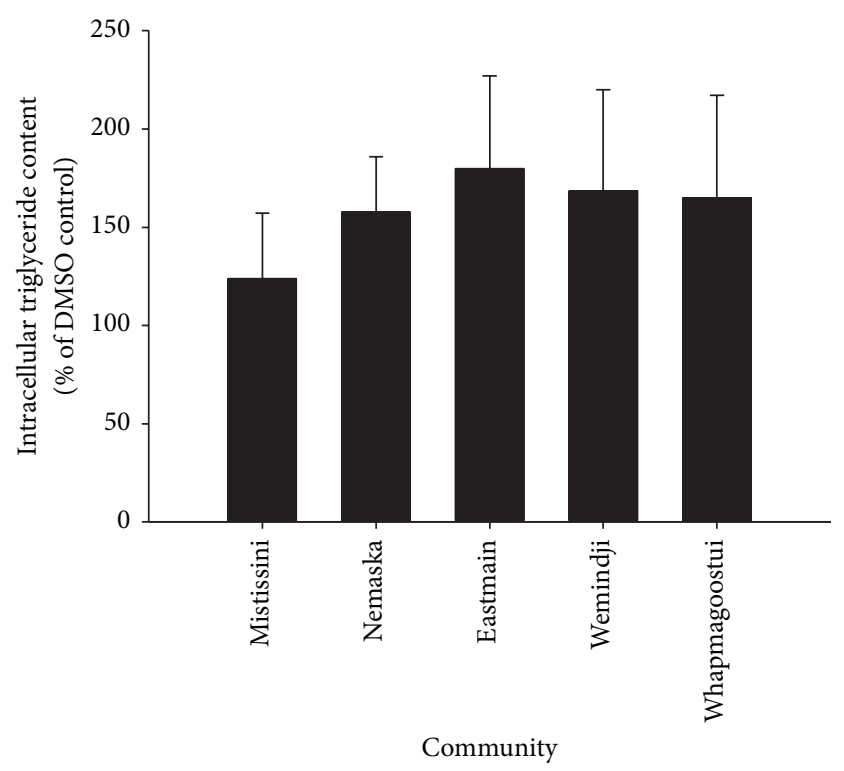

FIGURE 3: Effect of R. groenlandicum crude leaf extracts prepared from accessions collected around five communities in Northern Quebec on lipid accumulation. Intracellular triglyceride content was measured by AdipoRed fluorescence, in live 3T3-L1 murine adipocytes incubated with plant extracts for 5 days after differentiation. Means \pm SD $(n=5)$ are normalized to the vehicle control $(0.1 \%$ DMSO). There were no significant differences between communities $(p=0.348)$.

adipogenesis in a dose-dependent manner. The activity of quercetin is well studied and has also been consistently shown by others to be a potent inhibitor of adipocyte differentiation and adipogenesis [18, 32-34].

Our results suggest that while the geographical location does not appear to have a statistically significant impact on the adipogenic activity of crude extracts of localized R. groenlandicum samples, variations in active compounds do explain a significant proportion of variability in pharmacological activity. We have shown that annual temperature ranges and insolation parameters, such as solar radiation, could significantly explain some of the variation in the species' phenolic compounds [1]. Although we did not find in this study that these environmental variables could significantly explain the variation in TG content $\left(p=0.150, R_{\text {adj }}^{2}=0.162\right)$, we found, nonetheless, that they explained an important proportion of the variation in the phytochemical profiles of $R$. groenlandicum, which could significantly explain TG content (nontestable; see Table 1, Figure 6). Indeed, variation partitioning of TG content with both phytochemical and environmental variables indicates that while the relationship with compounds was statistically significant $(p=0.0279$, $\left.R_{\text {adj }}^{2}=0.491\right)$, their unique contribution to explaining TG content no longer was when the contribution of environmental variables, albeit small, was taken into account and removed $\left(p=0.0619, R_{\text {adj }}^{2}=0.424\right)$.

This confirms the caveat that environmental variables play an underlying role in affecting the content of biologically active compounds. Conversely, quercetin, the only significant 


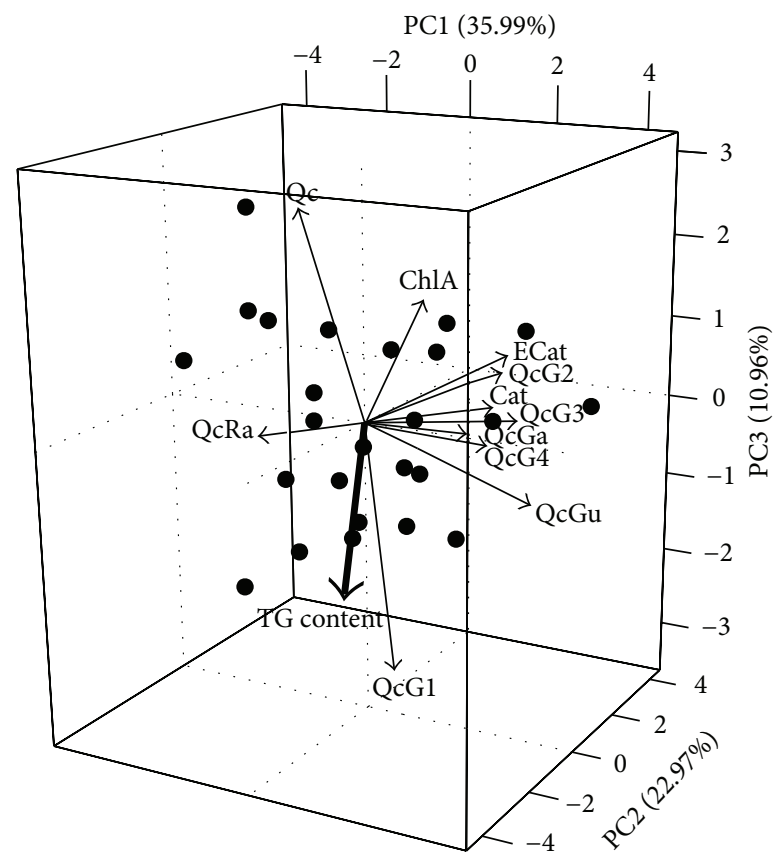

FIgURE 4: Principal component analysis biplot of 11 phenolic compounds in $R$. groenlandicum leaves. Solid lines represent relative loadings of these variables on axes 1,2 , and 3. TG content (bold arrow) was selected as a supplementary variable and plotted onto principal components generated from the phytochemical markers. Scores for individual samples are represented by symbols for

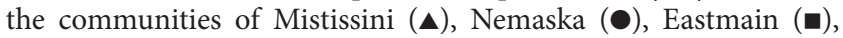
Wemindji $(\bullet)$, and Whapmagoostui $(\boldsymbol{\nabla})$. Abbreviations represent compounds as follows: (+)-catechin: Cat; chlorogenic acid: ChlA; (-)-epicatechin: ECat; quercetin-3-galactoside: QcGa; quercetin-3glucoside: QcGu; quercetin-glycoside 1: QcG1; quercetin-glycoside 2: QcG2; quercetin-glycoside 3: QcG3; quercetin-glycoside 4: QcG4; quercetin-3-rhamnoside: Qc-Ra; and quercetin: Qc.

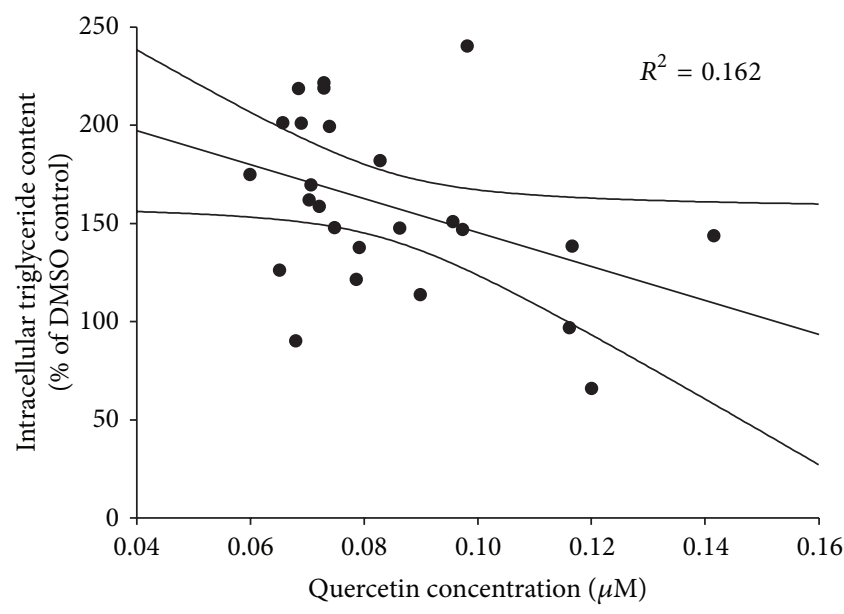

FIGURE 5: Intracellular triglycerides content of 3T3-L1 murine adipocytes exposed to $75 \mu \mathrm{g} / \mathrm{mL}$ of $R$. groenlandicum leaf extracts collected from various locations. Quercetin concentrations in crude extract were significantly and negatively associated with the species' adipogenic activity $(p=0.0458)$. Triglyceride contents are normalized to the vehicle control (0.1\% DMSO).
TABLE 1: Variation partitioning of the adipogenic activity of R. groenlandicum leaf extracts explained by the content in biologically active compounds (compounds) and the effect of bioclimatic variables (environment). Fraction $[a]$ corresponds to the unique contribution of compounds once the environment has been taken into account, whereas fraction $[c]$ represents the reverse. Fraction $[b]$ represents the shared portion, or overlap, between the effect of compounds and environment. The variation $\left(R_{\text {adj }}^{2}\right)$ of each fraction is represented in Figure 6. Asterisk $(*)$ indicates significant fractions at $\alpha=0.05$.

\begin{tabular}{lcc}
\hline Fractions & $R_{\mathrm{adj}}^{2}$ & $p$ \\
\hline$[a+b]=$ compounds & 0.491 & $0.0279^{*}$ \\
{$[b+c]=$ environment } & 0.109 & 0.150 \\
{$[a+b+c]=$ compounds +} & 0.534 & $0.0437^{*}$ \\
environment (full model) & 0.424 & 0.0619 \\
{$[a]=$ compounds|environment } & 0.0668 & Not testable \\
{$[b]=$ shared } & 0.0424 & 0.302 \\
{$[c]=$ environment|compounds } & 0.466 & Not testable \\
{$[d]=$ unexplained (residuals) } & &
\end{tabular}

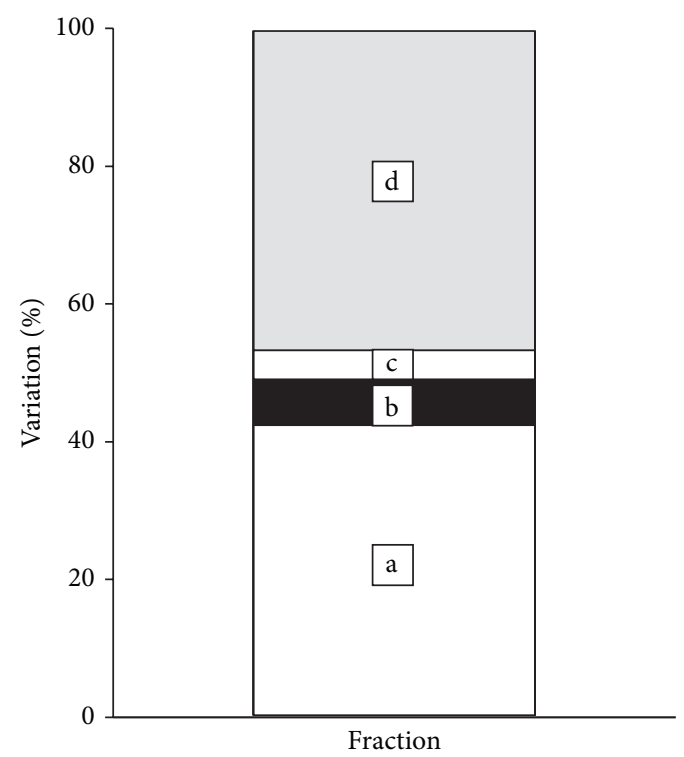

FIGURE 6: Variation partitioning of the adipogenic activity of $R$. groenlandicum leaf extracts explained by the content in biologically active compounds and the effect of bioclimatic variables. $a=$ compounds $\mid$ environment, $b=$ shared component, $c=$ environment|compounds, and $d=$ unexplained (see Table 1 for more details). The full model, fraction $[a+b+c]$, as well as the model including compounds only, fraction $[a+b]$, was significant at $\alpha=$ 0.05 .

compound related to changes in the adipogenic activity of $R$. groenlandicum, was not found to be strongly associated with environmental variables [1]. This may hence explain why the portion of variation those environmental variables contribute to the model, and more specifically to the content of biologically active compounds, which best explains the variation in TG content, is considerably small (Table 1, Figure 6).

On the other hand, our results provide support for the hypothesis that synergistic interactions may occur between 
compounds. For instance, the inhibitory action of Hibiscus sabdariffa (Malvaceae) was greater than the sum of its parts when polyphenols had been fractionated, isolated, and tested individually [35]. More importantly, in bioassay-guided fractionation experiments, the activity of crude Labrador tea extracts was higher than that of each active compound tested individually [24]. Finally, quercetin and resveratrol, together, decreased lipid accumulation considerably more than each of these used separately at the same dose [34].

Many of the compounds quantified in this paper have shown adipogenic activity in some form or another. Quercetin-3-glucoside has been found toxic to adipocytes at relatively low concentration $(50 \mu \mathrm{M})$ but was not found to affect adipogenic activity [33]. Quercetin-3-rhamnoside has been found inactive at low concentrations yet inhibited adipogenesis at high concentrations and chlorogenic acid has also been found to inhibit intracellular triglycerides accumulation [33]. Content variations of some of these, particularly $(+)$-catechin and (-)-epicatechin, have been explained by environmental variables [1]. Although the individual effect of these compounds was not detected in our study, it does not undermine the role they may play when found in a cocktail of substances.

\section{Conclusion}

We have previously shown that latitude acted as a marker for the impact of environmental variables on phytochemical concentrations [1]. Therefore, a trend could possibly exist between abiotic factors and concentration of targeted secondary metabolites, but a larger sample size might be needed to detect it. There may also be other environmental, climatic, and even biotic factors that were not taken into account, which explain the changes in quercetin content. These may better explain the ecophysiological processes affecting the antidiabetic potential of $R$. groenlandicum. Increase in the adipogenic potential of this traditional medicine was associated primarily with lower concentrations of quercetin, but the cause for its variation will require further investigation. Nonetheless, our results do not provide enough evidence to justify the idea that specific accessions of Labrador tea may have reproducibly better adipogenic potential than others along a latitudinal gradient. Conversely, our study implies that random harvesting of $R$. groenlandicum in the Eeyouch territory of Northern Quebec should not have a major impact on the quality of traditional preparations or NHPs made from this plant.

\section{Conflict of Interests}

The authors declare that there is no conflict of interests regarding the publication of this paper.

\section{Acknowledgments}

This work was supported by the Canadian Institutes of Health Research (CIHR) Team Grant (CTP-79855) to Pierre S. Haddad, J. T. Arnason, and Alain Cuerrier and discovery grant to J. T. Arnason as well as funding from the Natural Sciences and
Engineering Research Council (NSERC), Canada's Northern Internship program, and Network Environments for Aboriginal Health Research (NEAHR) to M. Rapinski. Special thanks are due to the Elders of the Eeyou Istchee Cree Nations of Mistissini, Nemaska, Waskaganish, Eastmain, Wemindji, and Whapmagoostui for sharing their traditional knowledge and allowing us to collect medicinal plants from their lands with the purpose of bridging indigenous knowledge and contemporary science. The authors also thank the Cree Board of Health and Social Services of James Bay for their constant support, as well as A. Léger, N. Roy, A. Downing, Y. Tendland, B. Walsh-Roussel, C. H Ta, D. Vallerand, N. Shang, and M. Ouchfoun for helping out with field and lab work. Special recognition is due to Jonathan Ferrier who also provided comments, ideas, and support. Finally, thanks are due to S. Daigle and P. Legendre for statistical advice.

\section{References}

[1] M. Rapinski, R. Liu, A. Saleem, J. T. Arnason, and A. Cuerrier, "Environmental trends in the variation of biologically active phenolic compounds in Labrador tea, Rhododendron groenlandicum, from northern Quebec, Canada," Botany, vol. 92, no. 11, pp. 783-794, 2014.

[2] T. Arnason, R. J. Hebda, and T. Johns, "Use of plants for food and medicine by Native Peoples of Eastern Canada," Canadian Journal of Botany, vol. 59, no. 11, pp. 2189-2325, 1981.

[3] R. A. Zieba, Healing and Healers among the Northern Cree, University of Manitoba, 1990.

[4] E. V. Siegfried, Ethnobotany of the Northern Cree of Wabascal Desmarais, University of Calgary, 1994.

[5] R. J. Marles, C. Clavelle, L. Monteleone et al., Aboriginal Plant Use in Canada's Northwest Boreal Forest, Natural Resources Canada, Edmonton, Canada, 2008.

[6] A. Cuerrier and Elders of Kangiqsujuaq, The Botanical Knowledge of the Inuit of Kangiqsujuaq, Avataq Cultural Institute, Inukjuak, Canada, 2011.

[7] A. Cuerrier and Elders of Kangiqsualujjuaq, The Botanical Knowledge of the Inuit of Kangiqsualujjuaq, Nunavik, Avataq Cultural Institute, Inukjuak, Canada, 2011.

[8] A. Cuerrier, Elders of Umiujaq, and Elders of Kuujjuarapik, The Botanical Knowledge of the Inuit of Umiujaq and Kuujjuarapik, Nunavik, Avataq Cultural Institute, Inukjuak, Canada, 2011.

[9] A. Cuerrier and L. Hermanutz, Our Plants... Our Land. Plants of Nain and Torngat Mountains Basecamp and Research Station (Nunatsiavut), Institut de Recherche en Biologie Végétale, Montreal, Canada; Memorial University of Newfoundland, St. John's, Canada, 2012.

[10] M.-H. Fraser, Ethnobotanical Investigation of Plants Used for the Treatment of type 2 Diabetes by Two Cree Communities in Québec: Quantitative Comparisons and Antioxidant Evaluation, McGill University, 2006.

[11] C. Leduc, J. Coonishish, P. Haddad, and A. Cuerrier, "Plants used by the Cree Nation of Eeyou Istchee (Quebec, Canada) for the treatment of diabetes: a novel approach in quantitative ethnobotany," Journal of Ethnopharmacology, vol. 105, no. 1-2, pp. 55-63, 2006.

[12] A. Downing, Inter and Intra-Specific Differences in Medicinal Plant Use for the Treatment of Type II Diabetes Symptoms by the Cree Elders of Eeyou Istchee (QC), Université de Montréal, 2010. 
[13] M. Rapinski, Ethnobotanique de la Nation crie d'Eeyou Istchee et variation géographique des plantes médicinales antidiabétiques, Université de Montréal, 2012.

[14] D. C. A. Spoor, L. C. Martineau, C. Leduc et al., "Selected plant species from the Cree pharmacopoeia of northern Quebec possess anti-diabetic potential," Canadian Journal of Physiology and Pharmacology, vol. 84, no. 8-9, pp. 847-858, 2006.

[15] M.-H. Fraser, A. Cuerrier, P. S. Haddad, J. T. Arnason, P. L. Owen, and T. Johns, "Medicinal plants of Cree communities (Québec, Canada): antioxidant activity of plants used to treat type 2 diabetes symptoms," Canadian Journal of Physiology and Pharmacology, vol. 85, no. 11, pp. 1200-1214, 2007.

[16] D. Harbilas, L. C. Martineau, C. S. Harris et al., "Evaluation of the antidiabetic potential of selected medicinal plant extracts from the Canadian boreal forest used to treat symptoms of diabetes: part II," Canadian Journal of Physiology and Pharmacology, vol. 87, no. 6, pp. 479-492, 2009.

[17] C. S. Harris, L.-P. Beaulieu, M.-H. Fraser et al., "Inhibition of advanced glycation end product formation by medicinal plant extracts correlates with phenolic metabolites and antioxidant activity," Planta Medica, vol. 77, no. 2, pp. 196-204, 2011.

[18] X.-K. Fang, J. Gao, and D.-N. Zhu, "Kaempferol and quercetin isolated from Euonymus alatus improve glucose uptake of 3T3L1 cells without adipogenesis activity," Life Sciences, vol. 82, no. 11-12, pp. 615-622, 2008.

[19] P. A. Grimaldi, "The roles of PPARs in adipocyte differentiation," Progress in Lipid Research, vol. 40, no. 4, pp. 269-281, 2001.

[20] J. B. Hansen and K. Kristiansen, "Regulatory circuits controlling white versus brown adipocyte differentiation," The Biochemical Journal, vol. 398, no. 2, pp. 153-168, 2006.

[21] O. A. MacDougald and S. Mandrup, "Adipogenesis: forces that tip the scales," Trends in Endocrinology \& Metabolism, vol. 13, no. 1, pp. 5-11, 2002.

[22] E. D. Rosen and O. A. MacDougald, "Adipocyte differentiation from the inside out," Nature Reviews Molecular Cell Biology, vol. 7, no. 12, pp. 885-896, 2006.

[23] A. Saleem, C. S. Harris, M. Asim et al., "A RP-HPLC-DADAPCI/MSD method for the characterisation of medicinal Ericaceae used by the Eeyou Istchee Cree First Nations," Phytochemical Analysis, vol. 21, no. 4, pp. 328-339, 2010.

[24] M. Ouchfoun, Validation des effets antidiabétiques de Rhododendron groenlandicum, une plante médicinale des Cri de la Baie James, dans le modèle in vitro et in vivo: élucidation des mécanismes d'action et identification des composés actifs, Université de Montréal, 2011.

[25] LAAS, GLFC, CFS et al., Selected Modeled Climate Data for Point Locations, LAAS, Sault Ste. Marie, Canada, 2014.

[26] D. W. McKenney, J. H. Pedlar, K. Lawrence, K. Campbell, and M. F. Hutchinson, "Beyond traditional hardiness zones: using climate envelopes to map plant range limits," BioScience, vol. 57, no. 11, pp. 929-937, 2007.

[27] D. McKenney, P. Papadopol, K. Lawrence et al., "Customized spatial climate models for Canada," 2007.

[28] S. Lê, J. Josse, and F. Husson, "FactoMineR: an R package for multivariate analysis," Journal of Statistical Software, vol. 25, no. 1, pp. 1-18, 2008.

[29] D. Borcard, P. Legendre, and P. Drapeau, "Partialling out the spatial component of ecological variation," Ecology, vol. 73, no. 3, pp. 1045-1055, 1992.

[30] A. Méot, P. Legendre, and D. Borcard, "Partialling out the spatial component of ecological variation: questions and propositions in the linear modelling framework," Environmental and Ecological Statistics, vol. 5, no. 1, pp. 1-27, 1998.

[31] R Core Team, R: A Language and Environment for Statistical Computing, 2014, http://www.r-project.org/.

[32] K. Iwashtta, K. Yamaki, and T. Tsushida, "Effect of flavonoids on the differentiation of 3T3-L1 adipocytes," Food Science and Technology Research, vol. 7, no. 2, pp. 154-160, 2001.

[33] C.-L. Hsu and G.-C. Yen, "Effects of flavonoids and phenolic acids on the inhibition of adipogenesis in 3T3-L1 adipocytes," Journal of Agricultural and Food Chemistry, vol. 55, no. 21, pp. 8404-8410, 2007.

[34] J.-Y. Yang, M. A. Della-Fera, S. Rayalam et al., "Enhanced inhibition of adipogenesis and induction of apoptosis in 3T3L1 adipocytes with combinations of resveratrol and quercetin," Life Sciences, vol. 82, no. 19-20, pp. 1032-1039, 2008.

[35] M. Herranz-López, S. Fernández-Arroyo, A. Pérez-Sanchez et al., "Synergism of plant-derived polyphenols in adipogenesis: perspectives and implications," Phytomedicine, vol. 19, no. 3-4, pp. 253-261, 2012. 


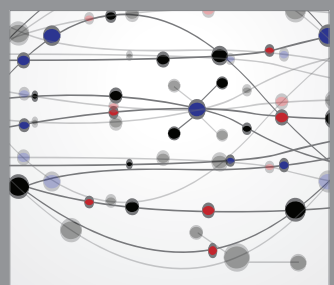

The Scientific World Journal
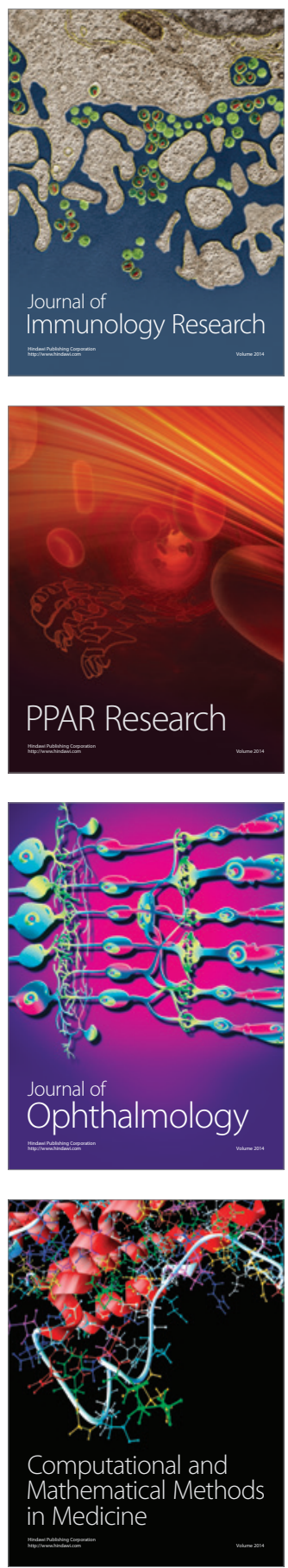

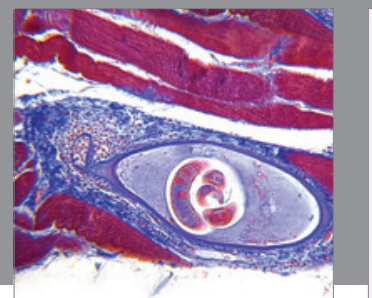

Gastroenterology

Research and Practice
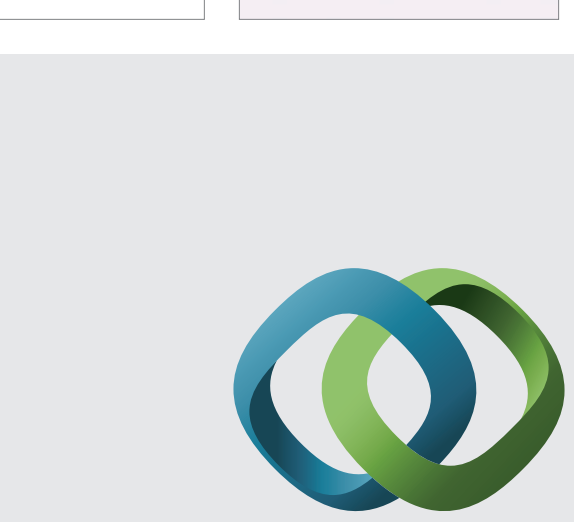

\section{Hindawi}

Submit your manuscripts at

http://www.hindawi.com
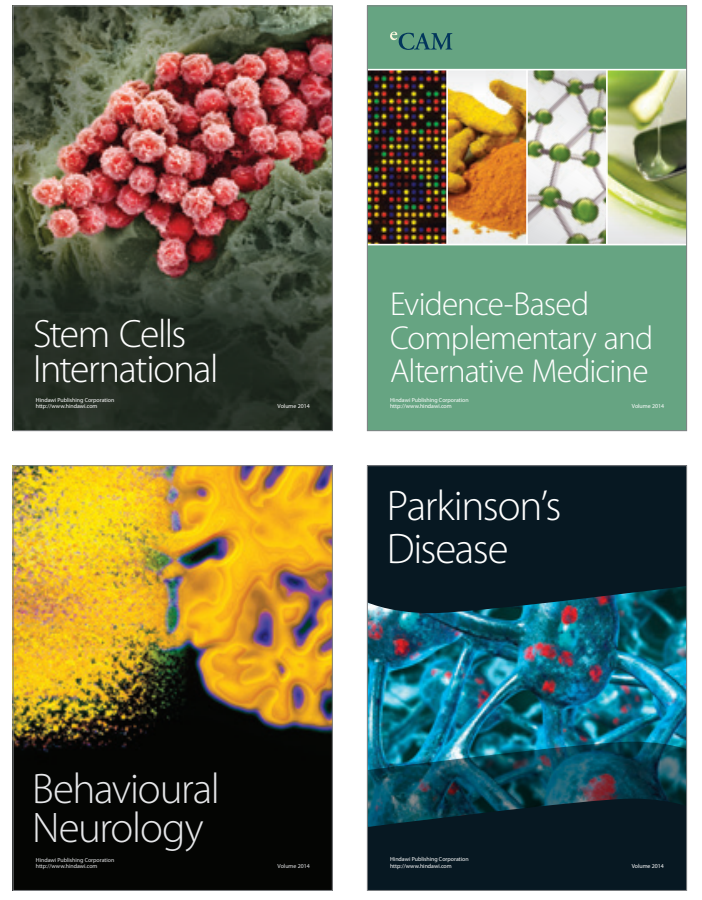
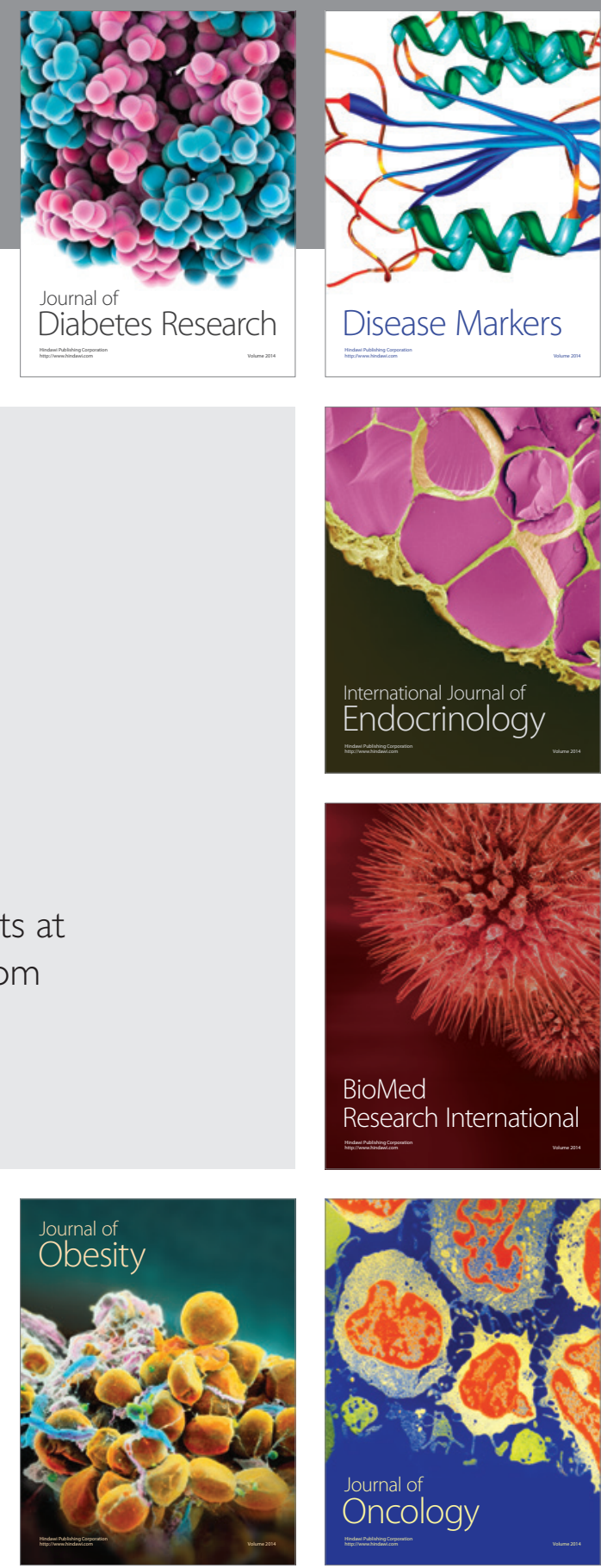

Disease Markers
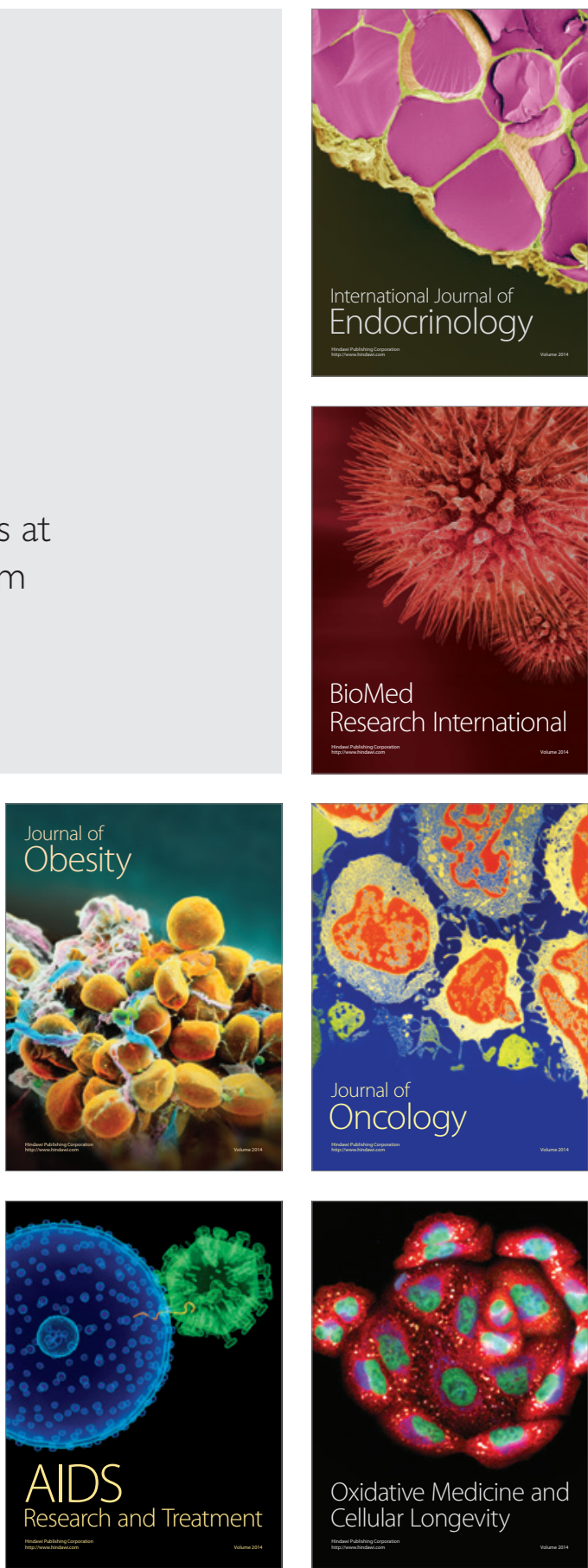